\title{
Modification of Post-Myocardial Infarction Granulocyte-Colony Stimulating Factor Therapy With Myelosuppressives
}

\author{
Yu Misao, MD; Masazumi Arai, MD; Takamasa Ohno, PhD; Hiroaki Ushikoshi, MD; \\ Hirohito Onogi, MD; Hiroyuki Kobayashi, MD; Genzou Takemura, MD; \\ Shinya Minatoguchi, MD; Takako Fujiwara, MD*; Hisayoshi Fujiwara, MD
}

\begin{abstract}
Background The purpose of the present study was to investigate the effect of granulocyte colony-stimulating factor (G-CSF) in combination with myelosuppressives on post-myocardial infarction (MI) myocardial repair. Methods and Results Twenty-four hours after 30-min ischemia and reperfusion (day 0), rabbits were assigned to 4 treatment groups: myelosuppressives (M group), G-CSF (G group), the 2 in combination (MG group) or saline (S group). Significantly greater numbers of circulating stem cells were seen in the MG group than in the $\mathrm{G}$ group, with attenuated leukocytosis. In addition, MG caused the greatest upregulation of stromal cell-derived factor (SDF)-1 within the infarcted myocardium and thus recruitment of stem cells from the circulation into the infarcted tissue. This led to enhanced myocardial repair, as indicated by the numbers of bone marrow cell-derived cardiomyocytes and endothelial cells, reduction in scar tissue, improvement in cardiac function and reduction in left ventricular remodeling during the chronic phase of MI. These beneficial effects were entirely abolished by the administration of a CXCR4 antagonist AMD3100, which indicates the importance of CXCR4/SDF-1-axis as a mechanism underlying myocardial repair.

Conclusion The combination of G-CSF and myelosuppressives may be a useful new therapy that overcomes the insufficiency seen with G-CSF alone. (Circ J 2007; 71: 580-590)
\end{abstract}

Key Words: Cardiac repair; CXCR4/SDF-1 axis; G-CSF; Myelosuppressives; Myocardial infarction

$\mathbf{T}$ he administration of granulocyte-colony stimulating factor (G-CSF) following myocardial infarction (MI) reportedly diminishes left ventricular (LV) remodeling and improves cardiac function via mechanisms that include myocardial regeneration and acceleration of the healing process, among others! ${ }^{-4}$ Underlying those beneficial effects is, mainly, the capacity of G-CSF to induce recruitment of bone marrow-derived stem/progenitor cells (BMSCs) to the infarcted myocardium. Nevertheless, recent clinical trials to investigate the post-MI administration of G-CSF have shown that the therapy led to no improvement in cardiac function and LV remodeling, although treatment with G-CSF is safe? Accordingly, we have been investigating alternative ways to use G-CSF, including in combination with other agents.

It is general knowledge in the fields of hematology and oncology that myelosuppressives such as 5-fluorouracil and cyclophosphamide mobilize BMSCs, and that this effect is augmented by adding G-CSF, 6,7 We previously

(Received October 16, 2006; revised manuscript received December 13, 2006; accepted January 23, 2007)

Department of Cardiology, Regeneration Medicine and Bioethics, Gifu University Graduate School of Medicine, Gifu, *Department of Food Science, Kyoto Women's University, Kyoto, Japan

Grant: Research Grants of Frontier Medicine (Nos. 15209027, 16590721), from the Ministry of Education, Culture, Sports, Science and Technology.

Mailing address: Hisayoshi Fujiwara, MD, Department of Cardiology and Regeneration Medicine and Bioethics, Gifu University Graduate School of Medicine, 1-1 Yanagido, Gifu 501-1194, Japan. E-mail: gifuim-gif@umin.ac.jp reported that post-MI treatment with myelosuppressives induces $\mathrm{CD} 4^{+}$cell mobilization via mild myelosuppression, reduces scar tissue and protects against LV remodeling? We therefore hypothesized that the administration of myelosuppressives prior to G-CSF amplifies the effects of G-CSF alone on BMSC recruitment and post-MI myocardial repair.

Stromal cell-derived factor (SDF)-1 expressed within the infarcted myocardium is well known to play a critical role in attracting mobilized CXCR4-positive BMSCs?,9 CXCR4+ cells contain not only CD34+ cells but also inflammatory cells such as monocytes/macrophages 10,11 and the cells mobilized via upregulation of the CXCR4/SDF-1 axis and recruited into infarcted myocardial tissue stimulate the post-MI healing process via paracrine effects and/or myocardial regeneration!,2 Bearing that in mind, we investigated the role played by the activated CXCR4/ SDF-1 axis in the beneficial effects of post-MI administration of myelosuppressives and G-CSF using the specific CXCR4 antagonist AMD3100, which disrupts recruitment of CXCR4+ cells, including stem cells and macrophages, into targeted tissues!

\section{Methods}

Rabbits used in the present study received humane care in accordance with the Guide for Care and Use of Laboratory Animals published by the US National Institutes of Health (NIH publication No. 83-23, revised 1996). The study protocol was approved by the Ethical Committee of Gifu University School of Medicine, Japan. 


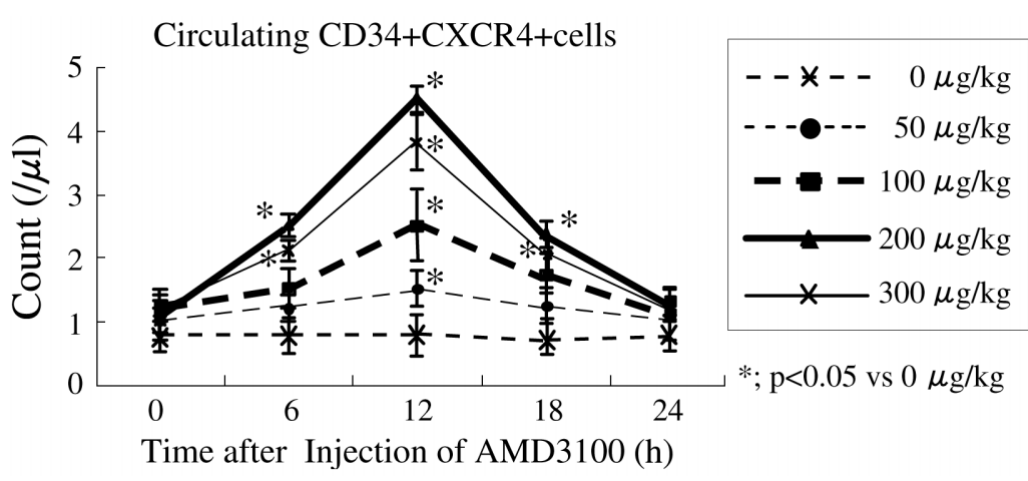

Fig 1. Characteristics of AMD3100. To determine an appropriate dosage of AMD3100 (a specific antagonist of CXCR4; octahydrochloride, Sigma) to block the CXCR4/stromal cell-derived factor-1 axis, normal rabbits were subcutaneously treated with 0 , $50,100,200$ or $300 \mu \mathrm{g} / \mathrm{kg}$ of AMD3100 $(\mathrm{n}=6$ in each). The $\mathrm{CD} 34{ }^{+} \mathrm{CXCR} 4+$ cells dose-dependently peaked at $12 \mathrm{~h}$ after the injection in rabbits receiving 50,100 or $200 \mu \mathrm{g} / \mathrm{kg}$, and dropped on the baseline at $24 \mathrm{~h}$ after injection. However, $300 \mu \mathrm{g} / \mathrm{kg}$ worked less effectively than $200 \mu \mathrm{g} / \mathrm{kg}$.
Ischemia-Reperfusion Infarct Model and Post-Reperfusion Treatment

Male Japanese white rabbits (approximately $2.0 \mathrm{~kg}$ ) were anesthetized by intravenous administration of $30 \mathrm{mg} / \mathrm{kg}$ sodium pentobarbital, after which a 30 -min ischemia and reperfusion protocol was carried out as previously described (day 0)! Briefly, under anesthesia and mechanical ventilation with room air, a left thoracotomy was performed, and 4-0 silk string was placed beneath the large coronary arterial branch coursing down the middle of the anterolateral surface of the LV. The 30-min coronary arterial occlusion and reperfusion was then performed by tightening and then releasing a snare made with the silk string. Rabbits surviving $24 \mathrm{~h}$ after reperfusion were randomly assigned to one of 8 groups ( $\mathrm{n}=20$ in each). Rabbits in the MG group were intravenously injected with 5 -fluorouracil $\left(5 \mathrm{mg} \cdot \mathrm{kg}^{-1} \cdot \mathrm{day}^{-1}\right)$ and cyclophosphamide $\left(20 \mathrm{mg} \cdot \mathrm{kg}^{-1} \cdot \mathrm{day}^{-1}\right)$ on days 1 and 2 ; G-CSF (G; $10 \mu \mathrm{g} \cdot \mathrm{kg}^{-1} \cdot \mathrm{day}^{-1}$ ) was subcutaneously injected on the following 5 days (days 3 to 7). Rabbits in the $M$ and $\mathrm{G}$ groups received either 5-fluorouracil/cyclophosphamide or G-CSF, respectively. Rabbits in the corresponding control (S) group received an appropriate volume of saline on the same days.

\section{Co-Administration of AMD3100 for Blockade of the CXCR4/SDF-1 Axis}

To determine an appropriate dosage of the specific CXCR4 antagonist AMD3100 (octahydrochloride, Sigma) with which to block the CXCR4/SDF-1 axis, normal rabbits were subcutaneously treated with $0,50,100,200$ or $300 \mu \mathrm{g} / \mathrm{kg}$ AMD3100 ( $\mathrm{n}=6$ in each). Their arterial blood was collected before treatment and 3, 6, 12, 18 or $24 \mathrm{~h}$ after treatment, and the $\mathrm{CD} 34{ }^{+} \mathrm{CXCR} 4{ }^{+}$cells were counted using an EPICS XL (Beckman Coulter). As shown in Fig 1, the numbers of $\mathrm{CD} 34^{+} \mathrm{CXCR} 4{ }^{+}$cells peaked dose-dependently $12 \mathrm{~h}$ after injection in rabbits receiving $0,50,100$ or $200 \mu \mathrm{g} / \mathrm{kg}$ AMD3100 and then returned to baseline within $24 \mathrm{~h}$. Because AMD3100 was less effective at a dosage of $300 \mu \mathrm{g} / \mathrm{kg}$ than at $200 \mu \mathrm{g} / \mathrm{kg}$, the latter was used to block the CXCR4/SDF-1 axis. Based on the data obtained, the remaining 80 rabbits, which were otherwise treated in the same manner as those in the MG, M, G and S groups, respectively, also simultaneously received the determined

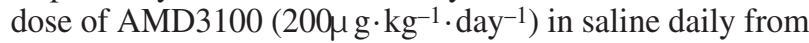
day 1 to 7 (AMG, AM, AG and AS groups; $n=20$, in each).

\section{Complete Blood Cell Counts (CBC) and $\mathrm{CD}^{2} 4^{+} \mathrm{CXCR} 4^{+}$ Cell Counts in Peripheral Blood}

Under anesthesia, arterial blood was collected from the ear arteries of rabbits in the MG, M, G and $S$ groups $(n=10$, in each) on days $0,3,7,14,21$ and 28 post-MI, and $\mathrm{CBC}$ and hemograms were obtained in co-operation with Mitsubishi Biochemical Laboratory (Japan).

For flow cytometric analysis, approximately $2.5 \mathrm{ml}$ of arterial blood were collected using a heparin sodium (500 U)loaded syringe, after which the mononuclear cell fraction was isolated on a ficoll gradient (JIMRO, Takasaki, Japan). After hemolysis of contaminating erythrocytes using ammonium chloride (Stem Cell Technologies), approximately $1.5 \times 10^{5}$ mononuclear cells were incubated with mouse anti-human FITC-conjugated CD34 monoclonal antibodies (mAb; Serotec) using the protocol provided by the manufacturer $\left(10 \mu 1\right.$ of mAb solution per $10^{6}$ cells in $100 \mu 1$ phosphate-buffered saline (PBS) for $60 \mathrm{~min}$ at room temperature) or with mouse anti-human PE-conjugated CXCR4 $\mathrm{mAb}$ (R\&D Systems) (10 1 per $10^{5}$ cells in $100 \mu 1$ PBS for $60 \mathrm{~min}$ at room temperature)?

\section{Echocardiography}

Prior to infarction and then 28 days post-MI, 10 rabbits in each group were placed under sodium pentobarbital $(30 \mathrm{mg} / \mathrm{kg})$ anesthesia and examined using transthoracic echocardiography (M-mode, Aloka SSD4000) with a $7.5-\mathrm{mHz}$ sector scan probe. The LV end-diastolic dimension (EDD, $\mathrm{mm}$ ) and ejection fraction (EF, \%) were measured.

\section{General Pathology}

Ten rabbits in each group were killed with an overdose of pentobarbital after heparinization $(500 \mathrm{U} / \mathrm{kg}) 7$ days postMI, and the remaining 10 in each group were killed 28 days post-MI. After measuring the body weight and LV weight, the LV was sliced into 7 transverse sections parallel to the atrioventricular ring from the apex to the base. All 7 sections from the hearts collected 28 days post-MI and the upper 2 sections from the center of the infarction in the hearts collected 7 days post-MI were fixed in $10 \%$ buffered formalin, embedded in paraffin, sliced into $4 \mu \mathrm{m}$-thick sections, and then stained with hematoxylin and eosin or Sirius red. Using 7 preparations collected 28 days post-MI, LV wall areas, infarct areas, surviving areas, and scar areas were calculated using a light microscope-connected image analyzer (LUZEX-F, NIRECO, Tokyo). Values are expressed as $\mathrm{mm}^{2} /$ slice and are mean values of 7 slices per heart.

\section{Immunohistochemistry}

Sections from the paraffin-embedded preparations described above were also used for immunohistochemistry. Using the indirect immunoperoxidase method, immunohistochemistry was carried out with mouse anti-human CXCR4 mAb (1:250, R\&D Systems), mouse anti-rabbit macrophage mAb (1:100, DAKO-RAM 11), mouse antihumana -smooth muscle actin mAb (1:250, DAKO), mouse 
A

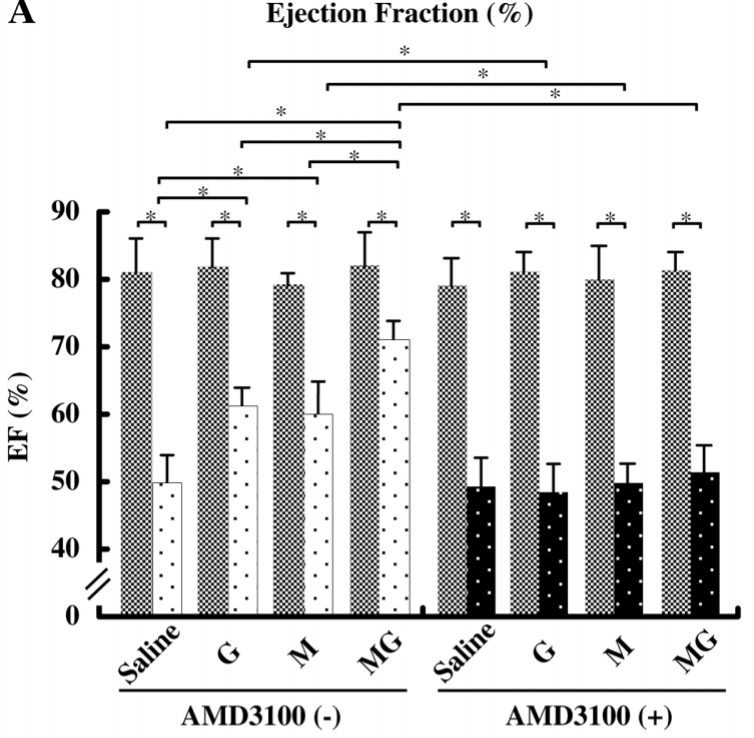

B

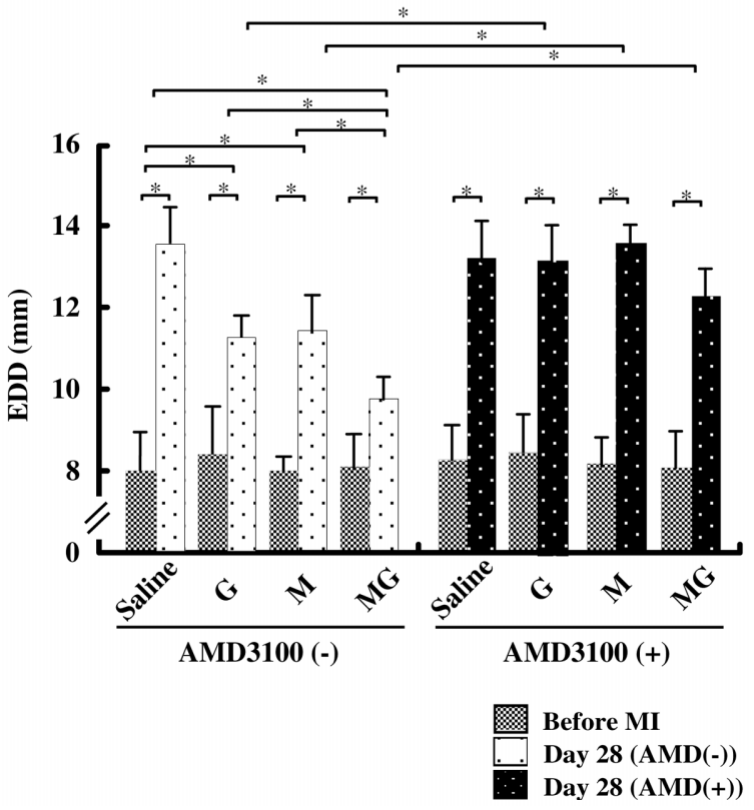

Fig 2. Echocardiography illustrating the effect of granulocyte-colony stimulating factor (G-CSF) and/or myelosuppressives on cardiac function and remodeling 28 days post-myocardial infarction (MI). Rabbits receiving G-CSF and/or myelosuppressives showed a significantly increased left ventricular ejection fraction $(\mathrm{EF})$ and a decreased left ventricular end-diastolic dimension (EDD), than the saline group. Note that the improvement in the cardiac function and remodeling was the greatest in the myelosuppressive-combined G-CSF group and the effects were inhibited by AMD3100.

anti-human endothelial cell CD31 mAb (1:100, DAKO) and mouse anti-human/mouse SDF-1 mAb (1:250, R\&D Systems) $)^{1,2}$

In Situ Detection of Nuclear DNA Fragmentation (TUNEL)

DNA fragments were labeled in deparaffinized $4 \mu \mathrm{m}$ thick sections from a transverse tissue block using an ApopTag in situ apoptosis detection kit (Chemicon-S7100), according to the manufacturer's instructions $!^{3}$
Western Blotting

Immediately after collecting and sectioning the hearts from rabbits killed 7 days post-MI, infarcted areas of the lowest 2 sections were snap-frozen for Western blotting. Fifty milligrams of frozen tissue from each heart were homogenized in lysis buffer and centrifuged for $10 \mathrm{~min}$ at $10,000 \mathrm{G}$ and $4^{\circ} \mathrm{C}$. Levels of SDF-1, vascular endothelial growth factor (VEGF) and matrix-metalloproteinase (MMP)1 were then assessed by Western blotting using mouse antihuman SDF-1 mAb (1:250, R\&D Systems), mouse antihuman VEGF mAb (1:250, Research Diagnostics), mouse anti-human MMP-1 mAb (1:500, Daiichi Fine Chemical), respectively, after which the signals were quantified by densitometry.

DiI-Labeling of Bone Marrow (BM)-Derived Mononuclear Cells and Laser Scanning Confocal Microscopy

In 48 rabbits under anesthesia, $10 \mathrm{ml}$ of iliac BM were aspirated and suspended in PBS containing 2,000 U of heparin sodium. BM-derived mononuclear cells (BMMNCs) were then isolated by centrifugation on a ficoll gradient, and approximately $1.0 \times 10^{8} \mathrm{BM}-\mathrm{MNCs}$ were suspended in $5 \mathrm{ml}$ of PBS. To label the cells 1,1'-dioctadecyl-1 to 3,3,3',3'-tetramethylindocarbocyanine-perchlorate dye (DiI, Molecular Probes) was added to the BM-MNCs, and then reinjected into the iliac bone! Thereafter, 6 rabbits in each group were subjected to the 30-min ischemia and reperfusion protocol. Twenty-eight days post-MI, the rabbits were killed and their hearts were immediately excised and placed in iced PBS. For immunohistocmemical analysis by confocal microscopy, samples (approximately $3 \times 3 \times 2 \mathrm{~mm}$ ) obtained from the center of the infarcted anterior LV wall were embedded in OCT compound (Miles Scientific), snap-frozen in liquid nitrogen and sliced into $6 \mu \mathrm{m}$-thick sections using a cryostat. Immunofluorescence microscopy (LSM510 NLO, Zeiss) was carried out using mouse antitroponin I mAb (1:5,000, Chemicon), mouse anti-human endothelial cell CD31 mAb (1:100, DAKO) and Hoechst 33342 for nuclear staining.

\section{Statistical Analysis}

All values are presented as the means \pm SD. Differences between groups were assessed using two-way repeated measures of analysis of variance with a post-hoc TukeyKramer's test. Values of $\mathrm{p}<0.05$ were considered to be significant.

\section{Results}

\section{Mortality}

All rabbits assigned to the MG, M, G, S, AMG, AM, AG or AS group $24 \mathrm{~h}$ after reperfusion survived the required 7 or 28 days.

\section{Echocardiography}

On day 28 post-MI, LVEF and LVEDD were significantly better in the MG group than in the G, M or S group, although the $\mathrm{G}$ and $\mathrm{M}$ groups showed significant improvement, as compared to the S group (Fig 2). These improvements were absent in the AMG, AM and AG groups, which also received AMD3100.

\section{CBC, Hemograms and $\mathrm{CD} 34^{+} \mathrm{CXCR} 4{ }^{+}$Cells in Peripheral Blood}

As shown in Fig 3, the leukocyte and granulocyte counts 
White Blood Cell

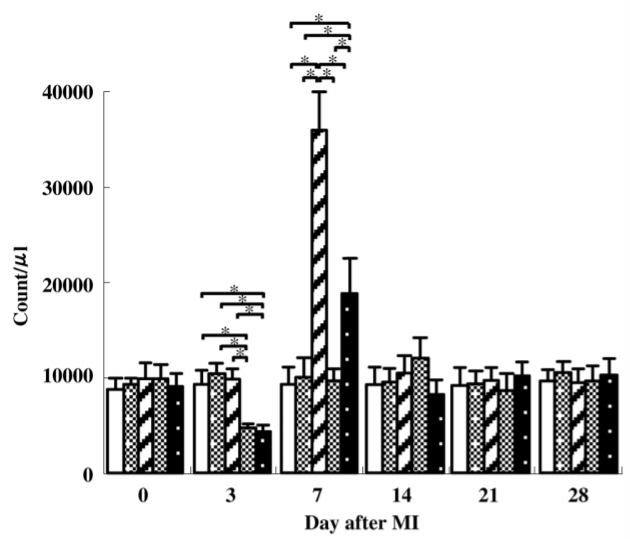

Monocytes

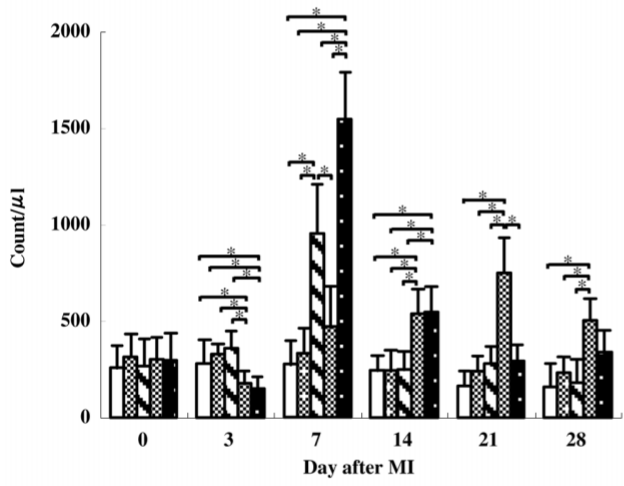

Circulating CD34(+)CXCR4(+) Cells

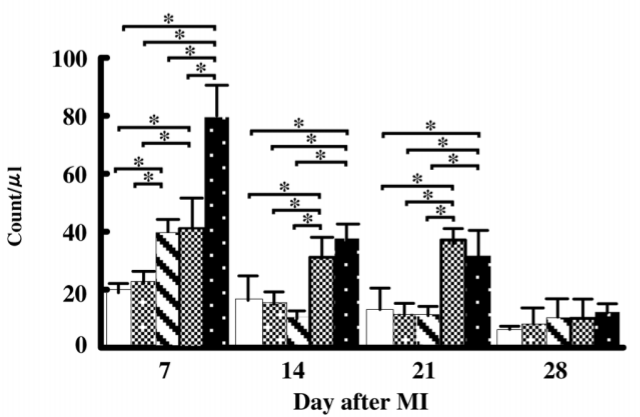

Granulocytes

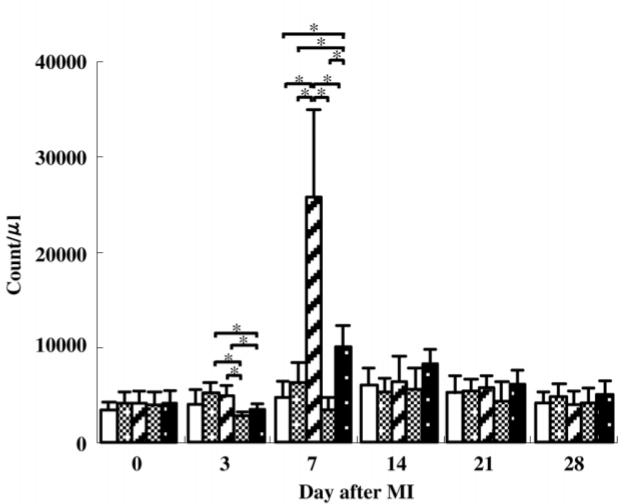

Lymphocytes

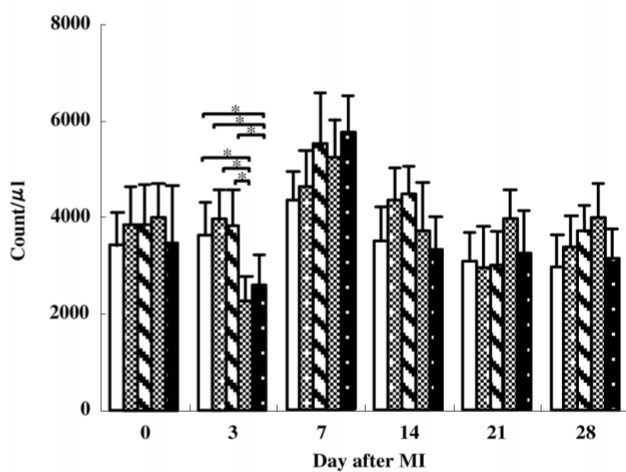

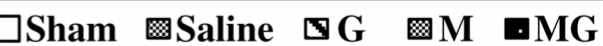

Fig 3. Peripheral blood cell counts (WBC). Granulocyte-colony stimulating factor (G-CSF) increased the number of WBCs and granulocytes 7 days after infarction, although the number of monocytes and circulating $\mathrm{CD} 34^{+} \mathrm{CXCR} 4^{+}$cells also increased in comparison with the saline group. In contrast, G-CSF with myelosuppressives further increased the number of circulating $\mathrm{CD} 34^{+} \mathrm{CXCR} 44^{+}$cells and monocytes in spite of the presence of leukocytosis attenuated by the preceding myelosuppression. MI, myocardial infarction.

were significantly higher in the $\mathrm{G}$ group than in any other group on day 7 post-MI. However, these increases were suppressed in the MG group. The numbers of monocytes and circulating $\mathrm{CD} 34{ }^{+} \mathrm{CXCR} 4{ }^{+}$progenitor cells were highest in the MG group. The treatment had no effect on either red blood cells or platelets (data not shown).

\section{General Pathology}

Seven days post-MI, the infarcted areas contained granulation tissue comprised of myofibroblasts and small vessels; scar tissue comprising collagen and fatty cells was seen 28 days post-MI. As shown in Fig 4, the transverse diameters of cardiomyocytes were significantly larger in the area bordering the infarction than in remote areas, and there was no significant difference among the 4 groups. In addition, the LV weights and LV wall areas were similar in the 4 groups 28 days post-MI; however, the infarct areas and the scar areas were the smallest in the MG group, and smaller in the $\mathrm{M}$ and $\mathrm{G}$ groups than in the $\mathrm{S}$ group. Conversely, the surviving areas were largest in the $\mathrm{MG}$ group and larger in the $M$ and $G$ groups than in the $S$ group. These effects were all eliminated with the administration of AMD3100.

\section{Immunohistochemistry}

The numbers of RAM $11^{+}$macrophages and CXCR4+ cells within the infarcted areas 7 days post-MI were significantly greater in the MG group than in the $\mathrm{M}$ and $\mathrm{G}$ groups, 
A Cardiomyocytes Transverse Diameter

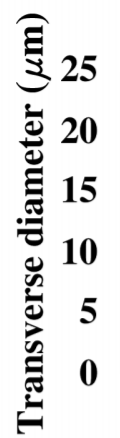

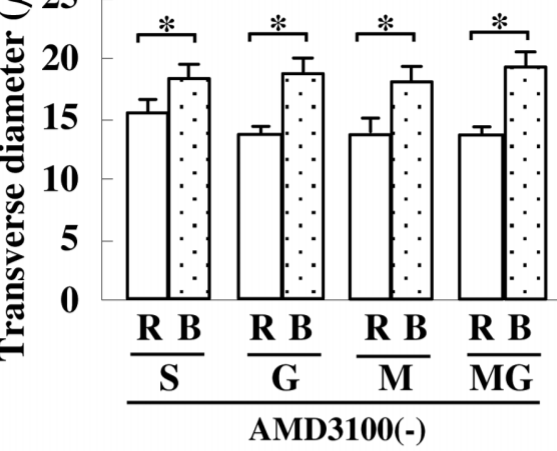

B

\section{Weight}
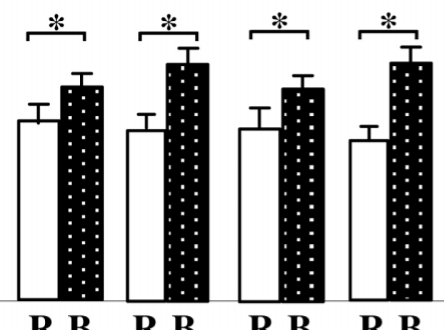

$\begin{array}{lllll}\frac{R \text { B }}{S} & \frac{R ~ B}{G} & \frac{R ~ B}{M} & \frac{R ~ B}{M G} & \begin{array}{l}\text { R; Remote area } \\ \text { B; Border area }\end{array}\end{array}$

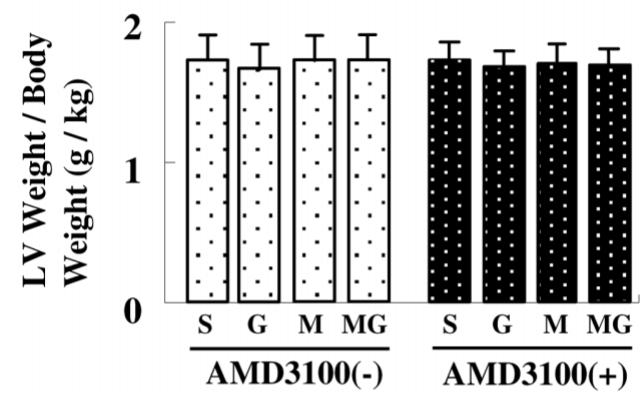

C

LV Wall Area

D

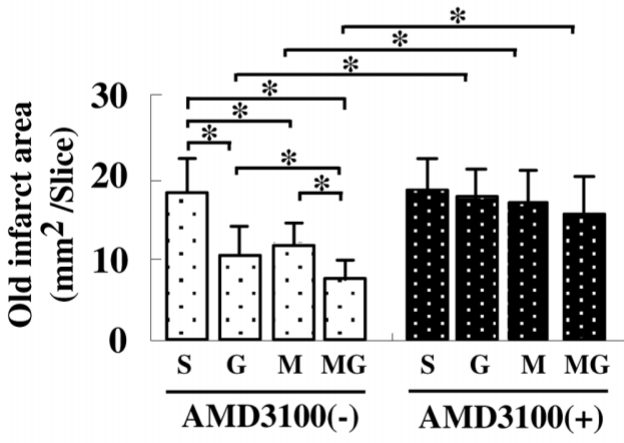

F
Infarct Area

\section{Scar Area (Fibrosis)}

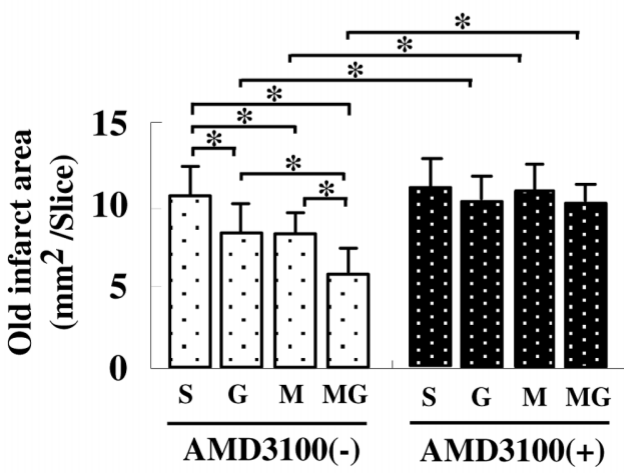

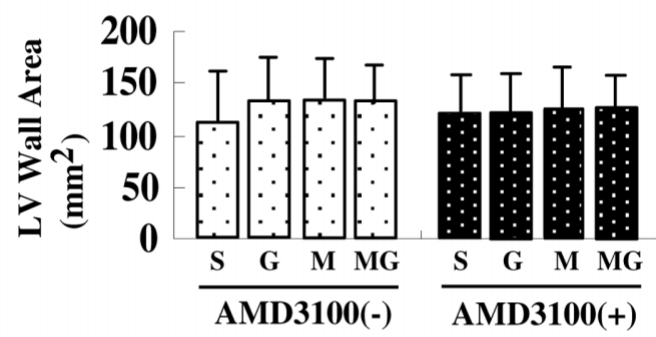

E

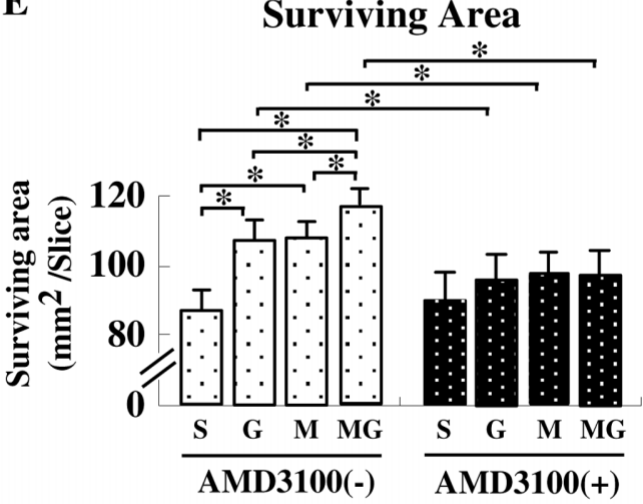

Fig 4. The transverse diameters of cardiomyocytes, left ventricular (LV) weight, LV wall area, infarct area, surviving area, and scar area 28 days post-myocardial infarction (MI). Granulocyte-colony stimulating factor (G-CSF) and/or myelosuppressives reduced the infarct areas and the scar areas, and induced a surviving myocardium, while the effect was the greatest in the G-CSF of the myelosuppressives group, and this effect was inhibited by AMD3100. There were no significant differences in LV weight, LV wall areas and cardiomyocyte transverse diameters among the groups. The border area was defined as areas of surviving myocardial tissue within $1 \mathrm{~mm}$ of the infarcted area. 
A RAM11(+) macrophages in infarct area

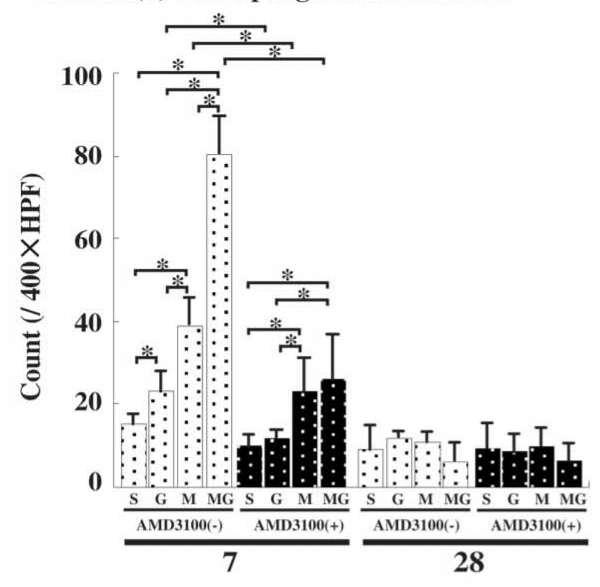

Day after Infarction
B

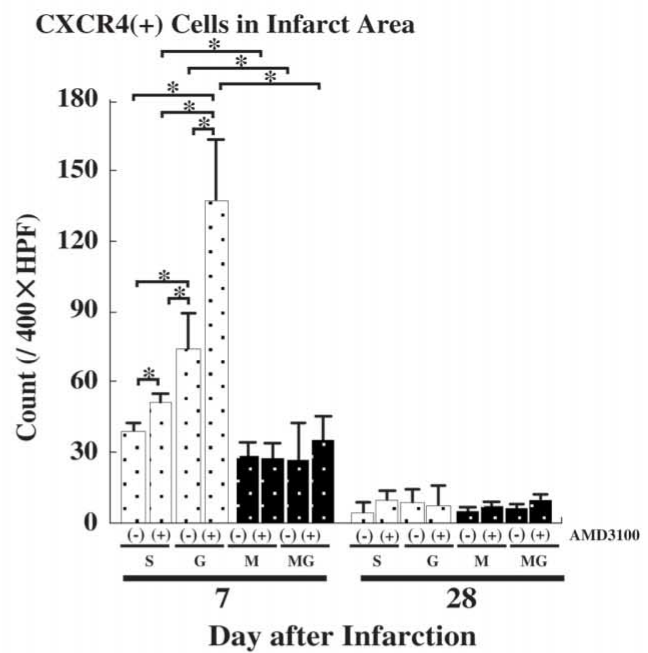

C $\mathrm{CD}^{+}{ }^{+}$-microvessels in peripheral infarct area
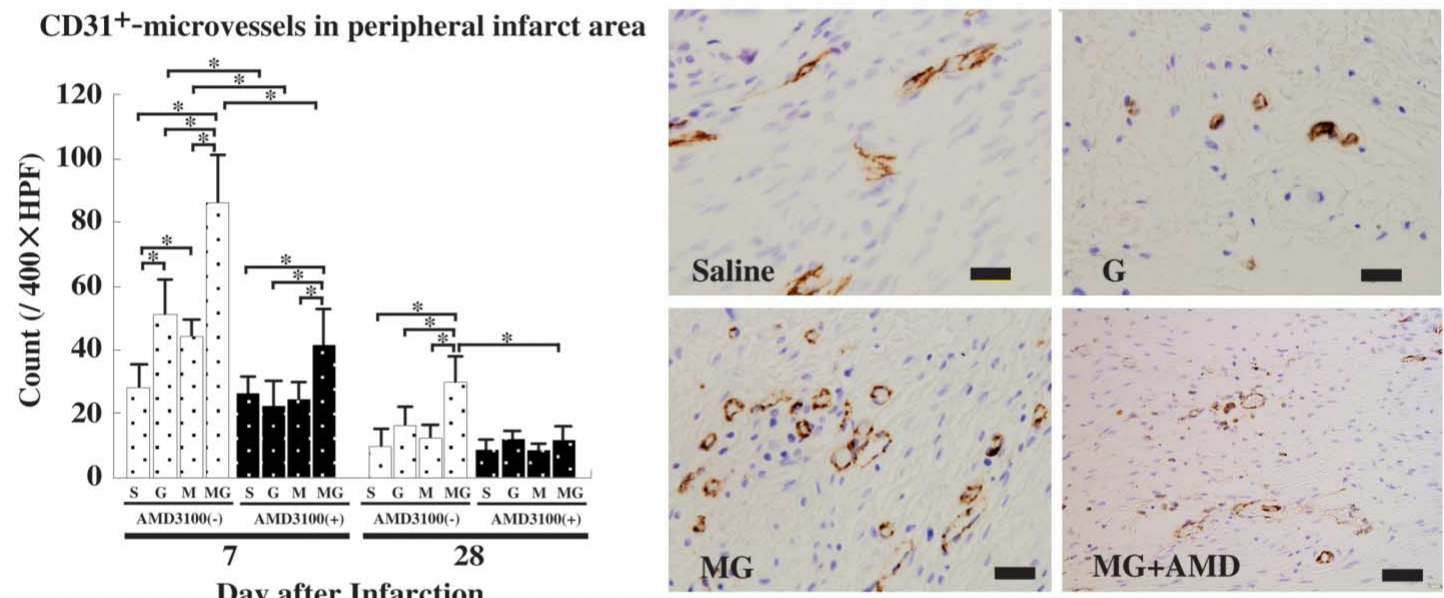

Day after Infarction

AMD;AMD3100, Bar ; $100 \mu \mathrm{m}$

D $\quad \alpha$-SM actin(+) myofibroblast - Day 28
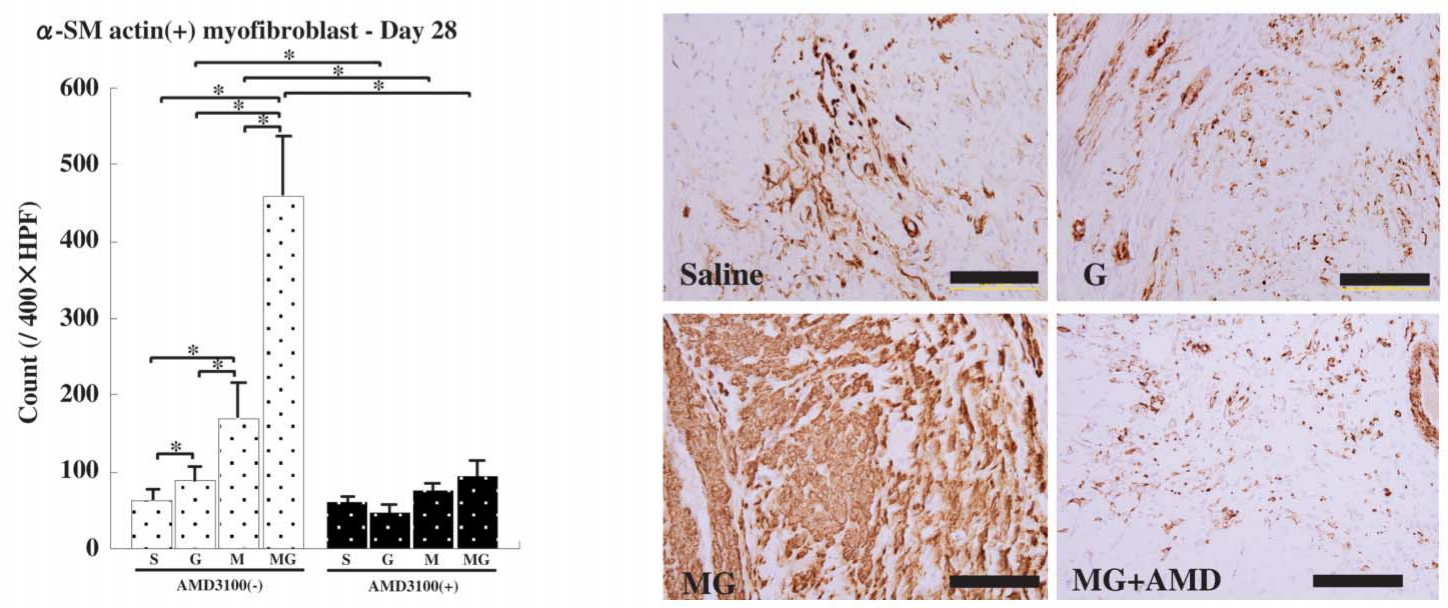

$\alpha$-SM actin(green); $\alpha$-smooth muscle actin, AMD;AMD3100, Bar ; $100 \mu \mathrm{m}$

Fig 5. An immunohistochemistry in the infarcted tissues. Recruitment of RAM11+ macrophages (A) and CXCR4+ cells (B) was induced by the granulocyte-colony stimulating factor (G-CSF) and/or myelosuppressives in comparison with the saline group, while G-CSF with myelosuppressives was found to be the most effective in the cell-recruiting effect. Increased counts ofa-smooth muscle (SM) actin ${ }^{+}$myofibroblasts and $\mathrm{CD} 31^{+}$endothelial cells within infarcted areas (per high power field) was seen in the treated groups, and furthermore, the combination therapy induced the greatest proliferation of microvessels and myofibrobrasts. Interestingly, the effects were inhibited by AMD3100. HPF, high power field. 
A

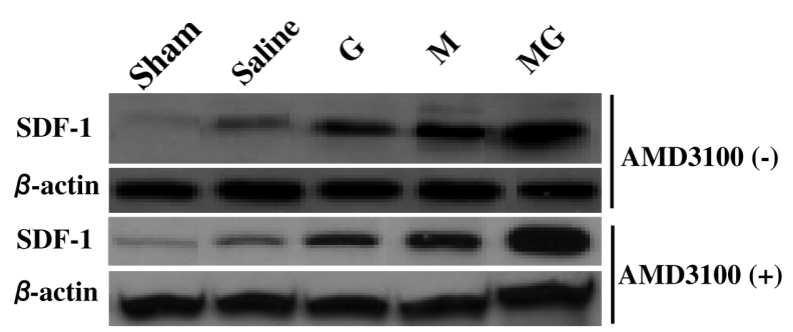

B

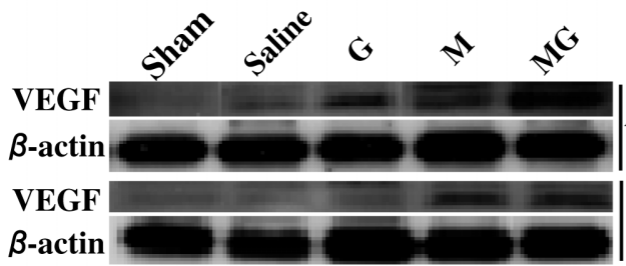

AMD3100 (-)

AMD3100 (+)

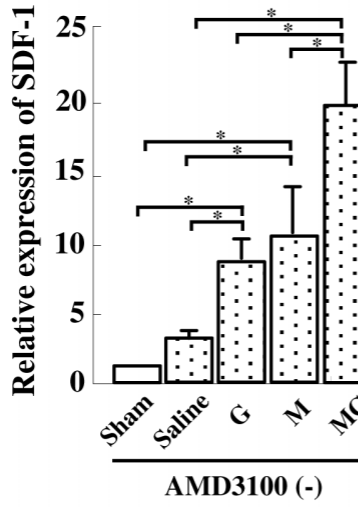

VEGF in infarcted myocardium (Western blot)

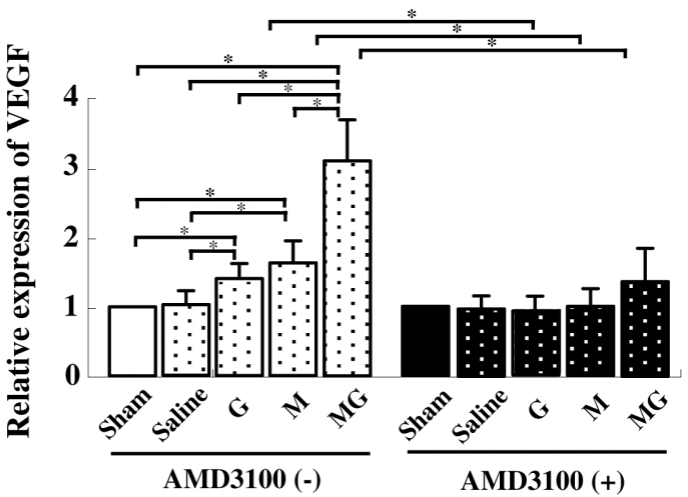

C

MMP-1 in infarcted myocardium (Western blot)

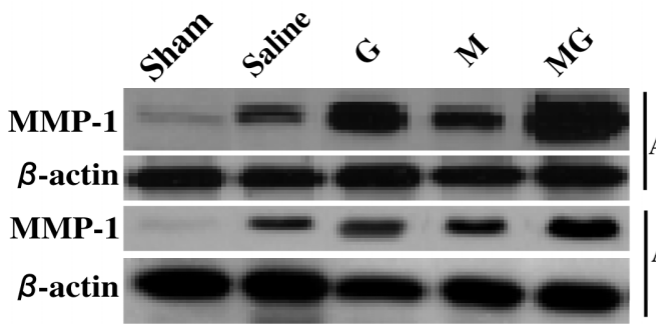

AMD3100 (-)

AMD3100 (+)

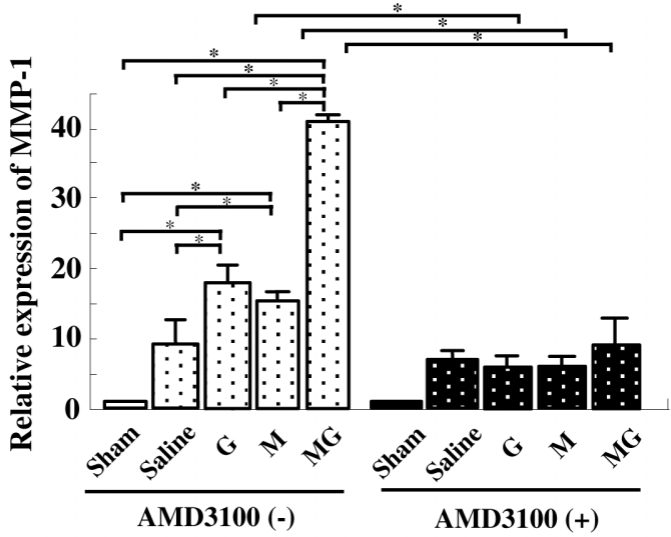

Fig 6. Western blot 7 days post-myocardial infarction. A greater upregulation of stromal cell-derived factor (SDF)-1, vascular endothelial growth factor (VEGF) and matrix-metalloproteinase (MMP)-1 in the left ventricular wall with infarction was induced by granulocyte-colony stimulating factor (G-CSF) with myelosuppressives than by any other treated groups, by G-CSF or myelosuppressives, which were all inhibited by AMD3100.

which were in turn greater than in the $\mathrm{S}$ group, but the increases were blocked by AMD3100 (Figs 5A,B). In addition, the numbers of $\mathrm{CD} 31^{+}$capillaries and $\mathrm{a}$-smooth muscle actin ${ }^{+}$myofibroblasts were greater in the infarcted areas of the MG group than in those of the other groups on day 28 post-MI. Again the increases were blocked by AMD3100.

\section{Western Blotting}

As shown in Fig 6, the expression of SDF-1, VEGF and
MMP-1 within the infarcted LV wall was significantly greater on day 7 post-MI in the MG group than in the M and $\mathrm{G}$ groups, which were in turn greater than in the $\mathrm{S}$ group. AMD3100 inhibited the upregulation of VEGF and MMP-1, but not the upregulation of SDF-1.

\section{BM Cell-derived Cardiomyocytes and Endothelial Cells}

The ratios of DiI-labeled cardiomyocytes to total cardiomyocytes and DiI-labeled endothelial cells to total endothelial cells in the areas bordering the infarction were 


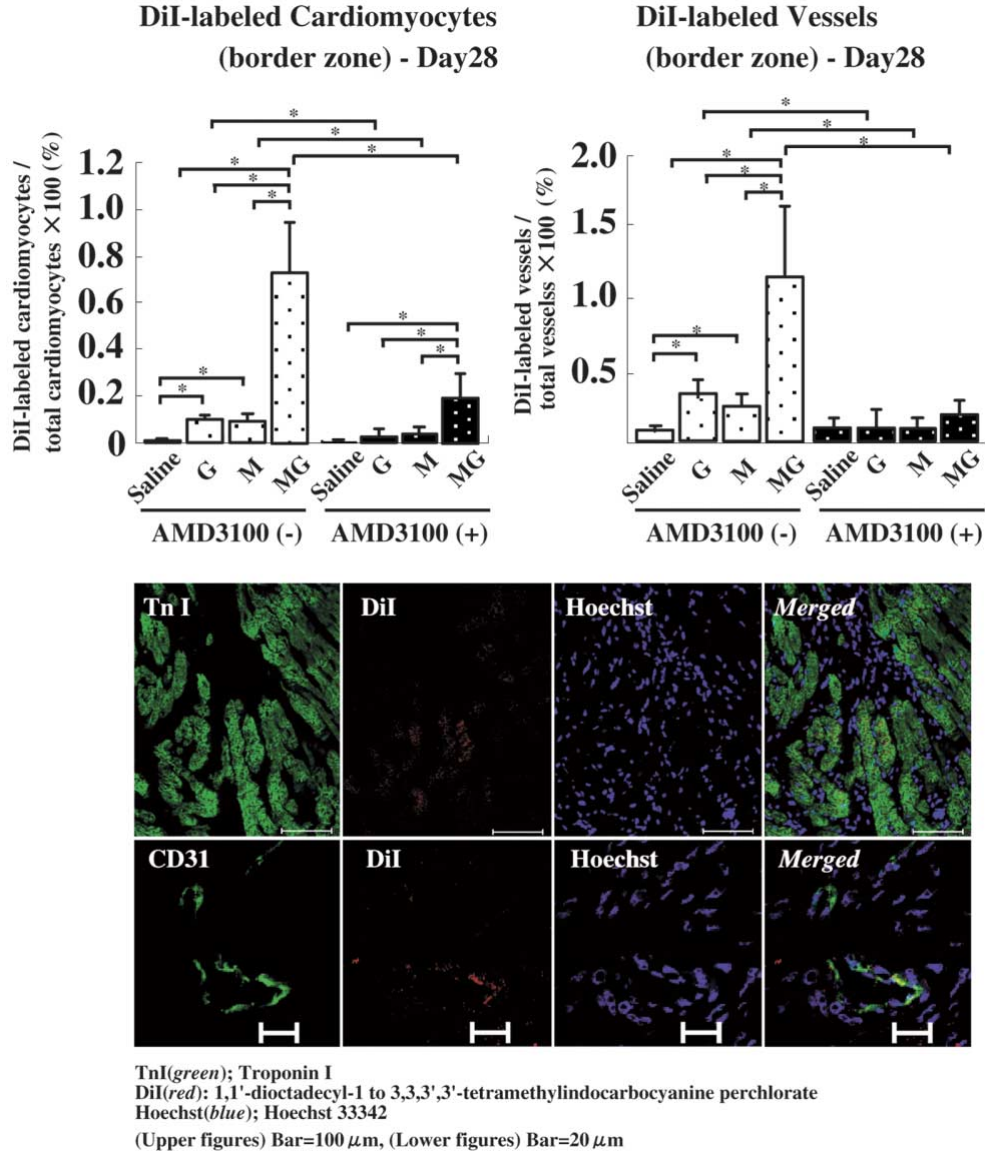

Fig 7. Bone marrow-derived mononuclear cell (BMMNC)-derived cardiomyocytes and endothelial cells detected by confocal microscopy 28 days post-myocardial infarction. Granulocyte-colony stimulating factor (G-CSF) and/or myelosuppressives increased the number of DiI-labeled-cardiomyocytes, thus suggesting the presence of BM-MNC-derived cardiomyocytes and endothelial cells, in the area bordering infarction, and note that G-CSF with myelosuppressives most effectively increased the cell number, and the effect was attenuated by AMD3100.
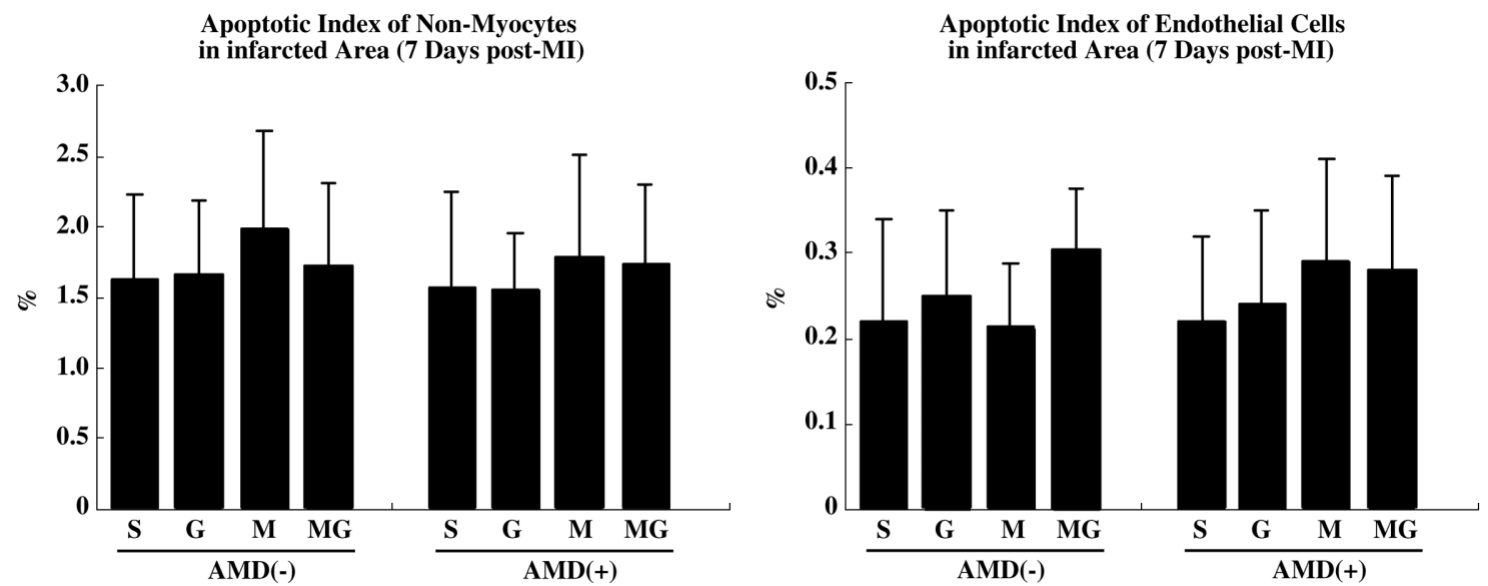

Fig 8. Apoptosis. Apoptotic indices of interstitial non-myocytes cells or endothelial cells within the infarcted area 7 days post-myocardial infarction (MI). Note that none of the therapies affected the apoptotic indices.

calculated for each group (Fig 7). Both of these ratios were significantly higher in the MG group than any of the other groups, and these increases were abolished in rabbits receiving AMD3100.

\section{Apoptosis}

Seven days post-MI, interstitial non-myocytes (eg, infiltrated inflammatory cells, endothelial cells) were often found to be TUNEL-positive; however, no TUNEL-positive cardiomyocytes were identified. As shown in the Fig 8, there were no significant differences in apoptotic indices among the treatment groups, which is consistent with our earlier findings $! 3,14$ In addition, co-administration of AMD3100 did not affect the incidence of apoptosis.

\section{Discussion}

Comparison of the Mechanisms Underlying the Beneficial Effects of G-CSF and Myelosuppressives

G-CSF and myelosuppressives show similar beneficial effects, as judged by their capacities to increase LVEF and diminish remodeling. The underlying mechanisms also are 
similar: they both reduce the size of old infarcts, increase the areas of surviving LV, increase the numbers of macrophages, $\mathrm{CXCR}^{+}{ }^{+}$cells and microvessels present, up-regulate VEGF and MMP-1, and, as herein shown, increase numbers of BM-derived cardiomyocytes and endothelial cells. That these beneficial effects were all inhibited by AMD3100, a specific CXCR4 antagonist, suggests they all depend on the recruitment of BM-derived cells into the myocardium.

As compared with myelosuppressives, G-CSF acts rapidly to induce cell migration by directly affecting CXCR4/SDF-1 signaling, but its effects quickly disappear, which is also shown in the present study! 15 By contrast, myelosuppressives first affect cell-cycling BMSCs then indirectly summon quiescent ones and activate them, eventually increasing the numbers of circulating BMSCs, so that the mobilization of $\mathrm{CD} 34{ }^{+} \mathrm{CXCR} 4{ }^{+}$cells into the peripheral circulation is sustained longer6 Consistent with those findings, we observed that the numbers of $\mathrm{CD}^{+} 4^{+}$ $\mathrm{CXCR}^{+}$cells in the peripheral circulation was elevated for a longer period in the $M$ group than in the $G$ group. Nevertheless, the efficiency by myelosuppressives was similar to that by G-CSF. This may be explained by 2 following possibilities. Firstly, the fact that upregulation of SDF-1 by both myelosuppressives and G-CSF, which is required to recruit circulating $\mathrm{BMSCs}$ into the myocardium, was limited to 7 days post-MI; thereafter, SDF-1 was no longer expressed within the infarcted myocardium (data not shown)? Consequently, even if a greater number of BMSCs are mobilized into the circulation by myelosuppressives, their recruitment into the myocardium may be limited by the availability of SDF-1. Second, unlike myelosuppressives, which are superior to G-CSF in the capacities to mobilize BMSCs, G-CSF has been reported to have an additional "cardioprotective" effect against ischemia-reperfusion injury! 16,17 As a result, they might seem similar in their apparent beneficial effects.

\section{Beneficial Effects of Post-MI Administration of G-CSF and Myelosuppressives}

Previous studies have shown that post-MI administration of G-CSF or myelosuppressives improves cardiac function and diminishes LV remodeling1,8 In the present study, the LVEF was greater and LVEDD was smaller in rabbits receiving the combination therapy than in those receiving either myelosuppressives or G-CSF alone. This suggests that the 2 agents act synergistically to improve cardiac structure and function following infarction. Moreover, according to Cesane et al, CD34+ cells mobilized by the combination of myelosuppressives and G-CSF would be functionally different from CD34+ cells mobilized by GCSF alone. In brief, CD34+ cells mobilized by the combination therapy have higher clonogenic activity and primitive progenitor cell content than $\mathrm{CD} 34^{+}$cells mobilized by G-CSF ${ }^{18}$

The leukocyte count is reportedly an independent risk factor and a prognostic factor associated with adverse outcomes in the clinical setting after MI because leukocytosis can lead to multiple adverse responses, including thrombosis among others.19 Post-MI administration of G-CSF causes a remarkable degree of leukocytosis, producing leukocyte counts of up to $40,000 \mu 1$, which could be a considerate issue in clinical settings. Likewise, in the present study, G-CSF treatment induced marked increases in the leukocyte and granulocyte counts by day 7 post-MI.
In contrast, myelosuppressives reduced the number of granulocytes, and in combination with G-CSF, they apparently inhibited the increases in the leukocyte and granulocyte counts seen with G-CSF alone.

\section{Possible Mechanism for the Improvement of Cardiac Structure and Function}

We suggest 2 possible mechanisms underlying the improvement in cardiac structure and function elicited by GCSF and myelosuppressives. First, the improvement in LV function may reflect repair-relating paracrine effects mediated by the cells recruited into the infarcted myocardium. The smaller scar tissue areas seen in the MG group likely contributed to the improved cardiac function and diminished remodeling. This would be explained by the significantly enhanced expression of MMP-1, which likely catalyzes the breakdown of collagen during the scar-formation process. Previous studies reported that an MMP family was increased in the post-MI heart failure models with permanent occlusion and large infarction, and the inhibitors beneficially affected cardiac remodeling and function, suggesting that an increase of MMP has a aggravating effect on heart failure via collagen degradation.20,21 In such cases with large infarction, collagen accumulation plays a critical role in preventing ventricular remodeling. In contrast, in the model of the present study, with moderate-sized infarction by a transient ischemia and reperfusion protocol, an excessive amount of fibrosis without contractility or relaxation would accelerate cardiac remodeling and decrease cardiac function, as seen in ischemic or idiopathic dilated cardiomyopathy, and accordingly, to maintain fibrosis to an adequate extent by an anti-fibrotic effect of MMP would be beneficial in preventing ventricular remodeling?22,23 Similarly, levels of the angiogenic growth factor VEGF were highest in the MG group, which may be related to the observed increase of microvessels. Marked proliferation of myofibroblasts containing large amounts of actin was also observed within the infarcted areas in the MG group. By protecting against wall stress, these cells also may play an important role in preventing ventricular remodeling? 4

The second is regeneration of myocardial tissue. The surviving LV area was greatest, and the numbers of BMderived cardiomyocyte-like cells within infarcted tissues were highest in the MG group, as a result of induced recruitment of $\mathrm{CD} 34{ }^{+} \mathrm{CXCR} 4{ }^{+}$cells, which is consistent with a previous report of dose-dependent CD34+-cell contribution to regeneration of infarcted myocardium 25 That all of these beneficial effects were blocked by AMD3100 is indicative of the importance of the paracrine effects and new tissue formation mediated by mobilized $\mathrm{CXCR} 4^{+}$cells within infarcted myocardial tissues. However, it is still unclear, as a limitation of the present study, whether the new myocytes and endothelial cells were formed by cell differentiation or by cell fusion.

A recent report has shown that G-CSF directly exerts an anti-apoptotic effect on myocardium following ischemia.26 In the present study, nevertheless, we detected no TUNELpositive cardiomyocytes in hearts from any of the groups, but only TUNEL-positive non-myocytes, including infiltrated inflammatory cells, endothelial cells and other interstitial cells, were without significant difference in all of the groups, suggesting that the therapeutic effects of G-CSF and myelosuppressives are unrelated to apoptosis. 


\section{No Serious Adverse Effect of Myelosuppressives}

The peripheral leukocyte counts were transiently reduced by myelosuppressives to approximately $5,000 \mu 1$ on day 3 post-MI after their administration, which was half the control level seen on day 0 , and completely recovered by day 7 . On days 7 and 28 post-MI, there was no difference in their survival or histological evidence of infectious disease in the hearts or lungs of the rabbits receiving myelosuppressives, as compared with rabbits not receiving myelosuppressives (data are not shown).

\section{Clinical Implications}

The efficacy of G-CSF alone for the treatment of MI remains controversial, as shown in the negative results of a recent double-blind controlled clinical trial5 As herein shown, the beneficial effects of G-CSF alone were significantly smaller than those elicited when G-CSF was administered in combination with myelosuppressives, which indicates there is room to improve G-CSF therapy. Accordingly, a modified G-CSF therapy, such as the one examined here, might be a more effective, novel strategy for enhancing post-MI myocardial repair in the clinical setting than GCSF alone. In fact, nevertheless, myelosupressives such as 5-fluorouracil and cyclophosphamide are potentially cardiotoxic. Five-fluorouracil gives rise to spasms of the coronary arteries, rhythm disturbance and myocardial and endothelial inflammation underlying their apoptosis at an acute phase of treatment, although at a higher dosage than in the present study. ${ }^{27}$ Similarly, a higher dose of cyclophosphamide is toxic for endothelium and myocytes, and causes interstitial hemorrhage and edema $2^{28}$ Although the dosage of the present experimental setting did not lead to any severe adverse effects, we need to monitor hemodynamics following treatment of the agents in the clinical setting closely. In conclusion, the combined administration of G-CSF and myelosuppressives following MI improves cardiac function and diminishes LV remodeling via increases in circulating CXCR4+ cells including CD $34^{+}$cells and repair-related inflammatory cells such as monocytes/macrophages, and may be a useful new therapy that overcomes the insufficiency seen with G-CSF alone.

\section{Acknowledgements}

We would like to thank Akiko Tsujimoto and the staff of Kyoto Women's University for their technical assistance. We also thank Mitsubishi Biochemical Laboratory for providing the complete blood cell counts and hemograms. The present study was supported in part by Research Grants of Frontier Medicine (Nos, 15209027, 15590732, and 14570700) from the Ministry of Education, Culture, Sports, Science and Technology.

\section{References}

1. Minatoguchi S, Takemura G, Chen X, Wang N, Uno Y, Koda M, et al. Acceleration of the healing process and myocardial regeneration may be important as a mechanism of improvement of cardiac function and remodeling by postinfarction granulocyte colony-stimulating factor treatment. Circulation 2004; 109: 2572-2580.

2. Misao Y, Takemura G, Arai M, Ohno T, Onogi H, Takahashi T, et al. Importance of recruitment of bone marrow-derived CXCR4(+) cells in post-infarct cardiac repair mediated by G-CSF. Cardiovasc Res 2006; 71: 455-465.

3. Valgimigli M, Rigolin GM, Cittanti C, Malagutti P, Curello S, Percoco $\mathrm{G}$, et al. Use of granulocyte-colony stimulating factor during acute myocardial infarction to enhance bone marrow stem cell mobilization in humans: Clinical and angiographic safety profile. Eur Heart J 2005; 26: $1838-1845$.

4. Suzuki K, Nagashima K, Arai M, Uno Y, Misao Y, Takemura G, et al. Effect of granulocyte colony-stimulating factor treatment at a low dose but for a long duration in patients with coronary heart disease:
A pilot study. Circ J 2006; 70: 430-437.

5. Ripa RS, Jorgensen E, Wang Y, Thune JJ, Nilsson JC, Sondergaard $\mathrm{L}$, et al. Stem cell mobilization induced by subcutaneous granulocyte-colony stimulating factor to improve cardiac regeneration after acute ST-elevation myocardial infarction: Result of the double-blind, randomized, placebo-controlled stem cells in myocardial infarction (STEMMI) trial. Circulation 2006; 113: 1983-1992.

6. Heissig B, Hattori K, Dias S, Friedrich M, Ferris B, Hackett NR, et al. Recruitment of stem and progenitor cells from the bone marrow niche requires MMP-9 mediated release of kit-ligand. Cell 2002; 109: $626-637$.

7. Jantunen E, Putkonen M, Nousiainen T, Pelliniemi TT, Mahlamaki E, Remes K. Low-dose or intermediate-dose cyclophosphamide plus granulocyte colony-stimulating factor for progenitor cell mobilisation in patients with multiple myeloma. Bone Marrow Transplant 2003; 31: 347-351.

8. Misao Y, Arai M, Ohno T, Ushikoshi H, Takahashi T, Takemura G, et al. Cyclophosphamide improves function of post-infarct hearts by reducing old infarct area and accelerating the mobilization of $\mathrm{CD} 34^{+}-$ cells. Circ J 2005; 69: 763-765.

9. Askari AT, Unzek S, Popovic ZB, Goldman CK, Forudi F, Kiedrowski M, et al. Effect of stromal-cell-derived factor 1 on stemcell homing and tissue regeneration in ischaemic cardiomyopathy. Lancet 2003; 62: 697-703.

10. Wojakowski W, Tendera M, Michalowska A, Majka M, Kucia M, Maslankiewicz K, et al. Mobilization of CD34/CXCR4 ${ }^{+}$, CD34/ CD117+, c-Met'stem cells, and mononuclear cells expressing early cardiac, muscle, and endothelial markers into peripheral blood in patients with acute myocardial infarction. Circulation 2005; 110: $3213-3220$.

11. Yi Y, Rana S, Turner JD, Gaddis N, Collman RG. CXCR-4 is expressed by primary macrophages and supports CCR5-independent infection by dual-tropic but not T-tropic isolates of human immunodeficiency virus type 1. J Virol 1998; 72: 772-777.

12. Hendrix CW, Flexner C, MacFarland R, Giandomenico C, Fuchs EJ, Redpath G, et al. Pharmacokinetics and safety of AMD-3100, a novel antagonist of the CXCR-4 chemokine receptor, in human volunteers. Antimicrob Agents Chemother 2000; 44: 1667-1673.

13. Takemura G, Ohno M, Hayakawa Y, Misao J, Kanoh M, Ohno A, et al. Role of apoptosis in the disappearance of infiltrated and proliferated interstitial cells after myocardial infarction. Circ Res 1998; 82: $1130-1138$.

14. Ohno M, Takemura G, Ohno A, Misao J, Hayakawa Y, Minatoguchi $\mathrm{S}$, et al. "Apoptotic" myocytes in infarct area in rabbit hearts may be oncotic myocytes with DNA fragmentation: Analysis by immunogold electron microscopy combined with In situ nick end-labeling. Circulation 1998; 98: 1422-1430.

15. Petit I, Szyper-Kravitz M, Nagler A, Lahav M, Peled A, Habler L, et al. G-CSF induces stem cell mobilization by decreasing bone marrow SDF-1 and up-regulating CXCR4. Nat Immunol 2002; 3: 687-694.

16. Takahama H, Minamino T, Hirata A, Ogai A, Asanuma H, Fujita M, et al. Granulocyte colony-stimulating factor mediates cardioprotection against ischemia/reperfusion injury via phosphatidylinositol3-kinase/Akt pathway in canine hearts. Cardiovasc Drugs Ther 2006; 20: $159-165$.

17. Ueda K, Takano H, Hasegawa H, Niitsuma Y, Qin Y, Ohtsuka M, et al. Granulocyte colony stimulating factor directly inhibits myocardial ischemia-reperfusion injury through Akt-endothelial NO synthase pathway. Arterioscler Throm Vasc Biol 2006; 26: 108-113.

18. Cesana C, Carlo-Stella C, Regazzi1 E, Garau D, Sammarelli G, Caramatti C, et al. CD34+ cells mobilized by cyclophosphamide and granulocyte colony-stimulating factor (G-CSF) are functionally different from CD34+ cells mobilized by G-CSF. Bone Marrow Transplant 1998; 21: $561-568$.

19. Madjid M, Awan I, Willerson JT, Casscells W. Leukocyte count and coronary heart disease. J Am Coll Cardiol 2004; 44: 1945-1956.

20. Creemers EE, Cleutjens JP, Smits JF, Daemen MJ. Matrix metalloproteinase inhibition after myocardial infarction: A new approach to prevent heart failure? Circ Res 2001; 89: 201-210.

21. Spinale, FG. Matrix metalloproteinases: Regulation and dysregulation in the failing heart. Circ Res 2002; 90: 520-530.

22. Heymans S, Luttun A, Nuyens D, Theilmeier G, Creemers E, Moons $\mathrm{L}$, et al. Inhibtion of plasminogen activators or matrix metalloproteinases prevents cardiac remodeling rupture but impairs therapeutic angiogenesis and causes cardiac failure. Nat Med 1999; 5: 11351142 .

23. Taniyama Y, Morishita R, Nakagami H, Moriguchi A, Sakonjo H, Shokei-Kim, et al. Potential contribution of a novel antifibrotic factor, hepatocyte growth factor, to prevention of myocardial fibrosis by angiotensin II blockade in cardiomyopathic hamsters. Circulation 
2000; 102: 246-252.

24. Hayakawa K, Takemura G, Kanoh M, Li Y, Koda M, Kawase Y, et al. Inhibition of granulation tissue cell apoptosis during the subacute stage of myocardial infarction improves cardiac remodeling and dysfunction at the chronic stage. Circulation 2003; 108: 104-109.

25. Iwasaki H, Kawamoto A, Ishikawa M, Oyamada A, Nakamori S, Nishimura $\mathrm{H}$, et al. Dose-dependent contribution of CD34-positive cell transplantation to concurrent vasculogenesis and cardiomyogenesis for functional regenerative recover after myocardial infarction. Circulation 2006; 113: 1311-1325.
26. Harada M, Qin Y, Takano H, Minamino T, Zou Y, Toko H, et al. GCSF prevents cardiac remodeling after myocardial infarction by activating the Jak-Stat pathway in cardiomyocytes. Nat Med 2005; 11: $305-311$.

27. Tsibiribi P, Bui-Xuan C, Bui-Xuan B, Lomberd-Bohas C, Duperret $\mathrm{S}$, Belkhiria $\mathrm{M}$, et al. Cardiac lesions induced by 5 -fluorouracil in the rabbit. Hum Exp Toxicol 2006; 25: 305-309.

28. Morandi P, Ruffini PA, Benvenuto GM, Raimondi R, Fosser V. Cardiac toxicity of high-dose chemotherapy. Bone Marrow Transplant 2005; 35: 323-334. 